
COPPER PLASMAS

AUThofis, Andrew D. Sappey, CLS-3

Thomas K. Gamble. CLS-3

SUamitted ro 22nd Fluid Dynamics, Plasma Dynamics and Lasers Conference June 24 through June 27, 1991

\title{
DISCLAIMER
}

This report wan prepared as an account of work aponsored by an agency of the United Siates Government. Neither the United States (jovvernment nor any agency thereof, nur uny of their employees, makes uny warraniy. express or implied, or annumes any lepul liabiluty ur reaponas bility for the accuracy. completenem, or usefulness of any informalium. apparatus, product. or process disclomed, or represents that its use would not infringc privately owned rights Refer ence herein (I) any specific commefcial product. procesen, or servic hy irade name, Irademark. manufacturer, or otherwine tioen not necemurily constilute or imply its endormement. recommendation, or favuring by the Inited Situtes (it, ernment or any agency thereul The views and opinions of authors expresuod herein do not nectenarily state or renect thime of the I niled Sitaten (bovernment or any apency therest.

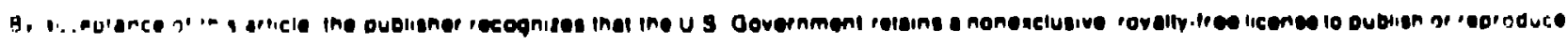

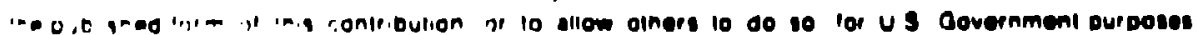

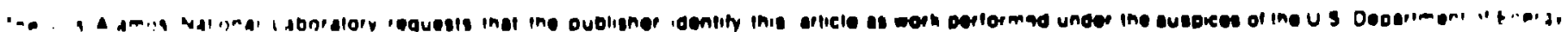

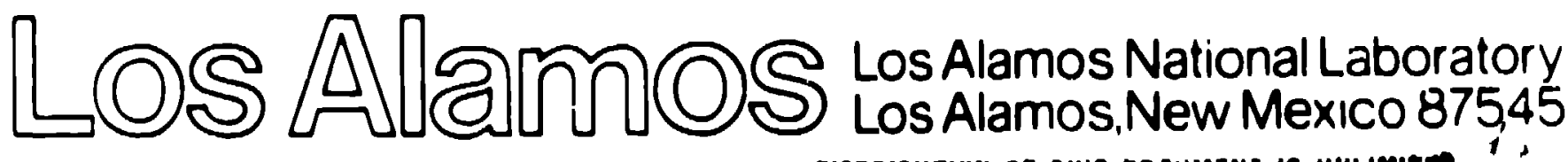


$\underline{\underline{\underline{\underline{E}}}}$

\section{AIAA $91-1460$}

Laser-Based Diagnostics for Condensation in Laser-Ablated Copper Plasmas

A. D. Sappey and T. K. Gamble Los Alamos National Laboratory Los Alamos, NM

AIAA 22nd Fluid Dynamics, Plasma Dynamics \& Lasers Conference

June 24-26, 1991 / Honolulu, Hawail 


\title{
LASER-BASED DIAGNOSTICS FOR CONDENSATION IN LASER-ABLATED COPPER PLASMAS
}

\author{
A. D. Sappey and T. K. Gamble \\ Chemical and Laser Sciences Division \\ Los Alamos National Laboratory \\ Los Alamos, New Mexico 87545
}

\begin{abstract}
Abstracs
We are investigating the thermodynamic conditions under which condensation occurs in laser-ablated copper plasrna plumes. The plasma is created by $\mathrm{XeCl}$ excimer laser ablation ( $308 \mathrm{~nm}, 350 \mathrm{~mJ} /$ pulse) at power densities from $5(0) 1000 \mathrm{MW} / \mathrm{cm}^{2}$. The atomic vapor expands rapidly into backing pressures of helium ranging from 0-50 tor. The backing gas serves to slow the vapor before it rarefies and provides a third body to stabilize collision complexes between vapor atoms to produce small cluster species. The ormation of these small clusters is indicative of the onset of condensation, a process which, under the proper conditions, eventually forms macroscopic particulate in the plume. We use laserinduced tluorescence (LIF) to probe both atomic cupper and the copper dimer molecule, $\mathrm{Cu}_{2}$. Velocities of atomic $\mathrm{Cu}$ have been obtained by a time-of-flight method under varying conditions of backing gas pressure. At low pressure (10 miorr), the atomic Cu velocity peaks at approximately $2 \times$ 1()$^{h} \mathrm{~cm} / \mathrm{s}$. Excitation scans of the $\mathrm{Cu}_{2} \mathrm{~A}-\mathrm{X}(0,0)$ and $(1,1)$ bands yield both a rotational temperalure and a vibrational temperature. Direct laser beam absorption is used to determine the number density of atomic copper. Rayleigh scattering from particulate is easily observable under condilions favorable to particulare production. The $\mathrm{Cu}_{2}$ I.IF and Rayleigh-scattered signals disappear instantaneously in the absence of the ablution tiser pulse indicating that the particulate is firmed during a single laser shot.

\section{Ineroduction}

L.aser ablation is a remarkably general method for provucing refractory muterials in the gas phase. Laser ablation sources are used in spectroscupic stucies of gas-phase metal clusters.' Commercial applications of laser ablation include the production of thin films of materials such as
\end{abstract}

superconductors ard fine metallic powc'ars. ${ }^{2,3}$ In the case of thin film production, it is desirable to avoid gas-phase condensation of the plume species which would produce inhomogeneities in the film, while condensation is necessary for the production of fine metallic powders. In both cases, knowledge of the conditions under which condensation takes place in the plume is of parilmount importance for understanding the conden. sation phenomenon and optimization of the process.

To date much of the present knowledge regarding condensation in laser-ablated plasmas is the result of work performed by Smalley and coworkers who developed a laser ablation source fior the pioduction and spectroscopic characterization of small metal clusters, $M_{n}\left(M_{n}, n\right.$ typically less than 200)." In this source, a laser beum is focused on to a metal rod that is rotating inside a holdur. The holder is attached to the end of a pulsed molecular beam valve. As the ablation laser is fired, the valve is opened allowing a high-density buffer gas pulse to entrain atomic material from the rod. The buffer gas and atomic species travel down a narrow channel (typically $1.3 \mathrm{~mm}$ in diameter). The buffer gas cools the ablated milterial and provides a third body to stabilize collision complexes between atomic and small molecular species to foster growth of the cluster species. The clusters expand into a vacuum at the end of the channel, which brings growth to an abrupt halt, and the resulting distribution of clusters mily be analyzed by mass or opeical spectrometric techniques. The distribution of clusters is found to be remarkably sensitive to both the pressure of the buffer gas pulse and the length of the chanmi-l. Higher backing pressures and longer challnels foster the growth of larger clusters."

Unfortunately, condensation in the Smalley source occurs inside the channel in a region thill 1. not easily accessed for diagnostic punposes 
making the study of such processes all but impossible. Variations of this source have been developed, but all share the same drawback.' Froben et al. claim to have developed a cluster source that has no channel. ${ }^{6}$ That is, the pulse from the supersonic valve expands freely to entrain the metal vapor. They offer an emission spectrum in the region of the $\mathrm{Cu}_{2} \mathrm{~A} \cdot \mathrm{X}$ transition as proof that the source produces clusters. However, we can assign every feature in their spectrum to $\mathrm{Cu}^{*}$. This certainly casts doubt on their conclusion.

Lyman has used laser ablation of various larget materials directly into a static background gis to produce macroscopic particulate. ${ }^{3}$ Particulate is generally formed only with backing gas pressures above $1-5$ torr. This is because at lower pressures. the atomic velocity is so high that the plume rarefies before a substantial number of collisions between plume species can occur. The background gas serves to slow and contain the atoms in a small enough volume that collisions between target material atoms can occur. In addition, the background gas provides a third body to stabilize collision complexes between atoms of the target material. This is necessary for small collision complexes, which do not have enough iniemal degrees of freedom to dissipate the hinding energy of the new atom. The geometry of this source is such that diagnostics are ciasily implemented. However, no in situ diagnostic's were performed in the previous studies to determine if the particulate is formed during 3 single shol or grows over a period of many "undreds of shots. We have adopted this expenmenial geometry and instituted in situ laser-based diagnostics 10 answer this question and to determine the thermodynamic conditions under which single shot particle production may occur.

\section{Experimental}

The experimental configuration is displayed in liig. I. Briefly, a 308-nm excimer laser beam (.3(X) $\mathrm{mJ}, 15 \mathrm{~ns}$ ) is focused by a $25-\mathrm{cm}$-focal length lens onto a rotalting copper target at normal incidence. The base pressure of the test chamber is $1(1)$ morr and the pressure can be increased from this value in approximately $8(X)$ torr with He huffer gas. During each experiment. a slow flow .: helium is maintained to minimize the build-up of background atomic $\mathrm{Cu}$ and particulate. The spot size at the target is approximately $0.1 \mathrm{~cm} \times$ $0.2 \mathrm{~cm}$. Copper is chosen as the target material for these studies because the spectroscopy of atomic $\mathrm{Cu}, \mathrm{Cu}_{2}$, and $\mathrm{Cu}_{3}$ is relatively well understood. At a variable time delay after the ablation laser pulse, an excimer pumped dye laser is fired. For $\mathrm{Cu}$ atom detection, the fundamental beam at $\sim 650 \mathrm{~nm}$ is frequency doubled in a KDP crystal to produce radiation in the vicinity of $325 \mathrm{~nm}$. This beam passes through the apparatus parallel to the copper target and perpendicular to the ablation laser beam. Fluorescence is collected along the third axis, which is perpendicular to both the ablation laser and the probe laser by a single 8.5 . $\mathrm{cm}$ focal length, $5-\mathrm{cm}$-diameter lens and focused on to the entrance slit of a $0.22-\mathrm{m}$ monochromator. The slits of the monochromator are typically. $0.1 \mathrm{~mm}$ wide and the height is approximately $2 \mathrm{~mm}$ giving a spectral resolution of $0.37 \mathrm{~nm}$. The distance from the target to the probe laser beam and detection system is variable so that the plume species can be probed as a function of distance from the target as well as delay time. This allows us to determine time-of-flight protiles and velocities for all species that can be detected by LIF.

For absolute calibration of the LIF signals. direct absorption experiments are pertionmed. In these experiments, the probe laser beam exiting the chamber impirges on a fluorescent card. 'The intensity of this fluorescence is monitored by a photomultiplier equipped with a neutral densi:y filter. An absorption experiment then consists of scanning the due laser frequency over a $\mathrm{Cu}$ atomic transition and observing the decrease in transmitted laser intensity. With knowledge of the dominant broardening mechanism and the degree of laser saturation, one can ralculare in effective cross section and therefore a $\mathrm{Cu}$ number density.

The $\mathrm{Cu}_{2}$ molecule has also been derected by LIF via the $A-X(0,0)$ transition. To our knowledge, this is the first observation of $\mathrm{Cu}_{2}$ produced in our experimentsl configuration. It has been pratuced previously in the Smalley source ${ }^{4}$ and in sputtering sources. ${ }^{7}$ The bandhead of this trills.i. iton ociurs at $\sim 490.27 \mathrm{~nm}$. We typically detect nuorescence on the ( $(1,1)$ band at $496.7 \mathrm{~nm}(1)$ avoid contamination of the fluorescence signill l! 
Rayleigh scattering from the particulate. This electronic system has been studied by a number of groups, but most recently by Page et al.' The specuroscopic constants have been determined accurately so that spectral simulations of the $(0,0)$ band can be computed at various temperatures and compared to experimental spectra to determine rotational and vibrational temperanures. Time-of-flight profiles can be obtained for $\mathrm{Cu}_{2}$ in the same manner as described above for $\mathrm{Cu}$.

In addition, we observe a strong Rayleigh scattered signal from the particulate at $90^{\circ}$ to the probe beim using the monochromator/phototube as the detector. This signal comprises an unwanted interference when attempting to detect resonance tluorescence, but it is extremely useful for determining where particulate is formed in the plume. Typically, we use LIF detection schemes, which observe fluorescence transitions to levels other than the ground stiste to avoid this problem. Contaminution of the Rayleigh scattered signal by LIF is avoided by performing the scattering experiments off of atomic or molecular resonances.

\section{Results and Discussion}

\section{Cu Atom LIF and Absorption Diagnostics}

\section{Cu Atum Laser-Induced Fluorescence}

Figure 2 displays an excitation scan of the $\cdot P_{1}<-S$ trunsitions in copper atom as well as an abridged Cu energy level diagram as an inset. We excite from the ground state to the two spin-orbit levels of the ${ }^{2} \mathrm{P}$ stite at the energies (wavelengths), $3\left(15.35 .3() 2 \mathrm{~cm}^{\prime}(324.754 \mathrm{~nm})\right.$ and $30783.686 \mathrm{~cm}^{-1}$ $(327.396 \mathrm{~nm})$, respectively. For this scan, fluorescence is detected at $578.4 \mathrm{~nm}$ to the ${ }^{2} \mathrm{D}$ level. The fact that we observe hoth ${ }^{2} \mathrm{P}$, components in the excitation scan, even though fluorescence is detecled from only the lower energy ${ }^{2} P$, compon'nt, is indicative that energy transfer occurs in the $: P$, manifold on a time scale competitive with the nuorescence lifetime $(-10 \mathrm{~ns})$ under these experimental conditions (5-tcrm He background gits). This is not particularly surprising since the iepirution between the ${ }^{2} \mathrm{P}$, components is only $-2(x) \mathrm{cm}$ '.
Figure 3 shows a dispersed fluorescence scan obtained by exciting the ${ }^{2} \mathrm{P}$, spin-orvit level of $\mathrm{Cu}$ atom at $324.754 \mathrm{~nm}$. Fluorescence bands ase observed at 324.8, 327.4.510.7, 570.2, and $578.4 \mathrm{~nm}$. If energy transfer between the spinorbit levels of the ${ }^{2} \mathrm{P}$, did not occur, one would expect fluorescence at $324.8,510 . ?$, and $570.2 \mathrm{~nm}$ only. Strong resonance fluorescence is observed: however, to avoid possible contamination of the LIF signal by Rayleigh scattering from particulate, we iypically observe fluorescence to the $2 D$ spin 'urbit levels. It should be noted that the 'P and ${ }^{2} \mathrm{D}$ levels are the lasing levels of the copper vapor laser. In fact, under some circumstances, we observe stimulated emission (bi-directional lasing action) on the 510.7-nm transition, which i, easily visible by eye.

\section{Cu Number Density from Absorption Measurements}

In order to quantify the number density of $\mathrm{Cu}$ under various backing pressure conditions, we have performed direct laser beam absorption experiments. Equation (1) gives a general expression for the transmitted intensity in an absorption experiment where i denotes the lower energy levil and $k$ the excited level; $\alpha_{i j}(v)$ is the frequency dependent absorpion coefficient.'

$$
\begin{aligned}
d I & =-\iint \alpha_{\mathrm{u}}(v) I(v) d v d z \\
\alpha_{i}(v) & =\alpha_{0} g\left(v-v_{0}\right)
\end{aligned}
$$

For situations in which the linewidth of the luser is less than that of the absorbing transition. $I(v)$ may not be taken out of the integral. In our ex. periment, the width of the absorption lines are typically $1 \mathrm{~cm}^{\prime}$. while the bandwidth of the frequency-doubled probe laser is $0.1-0.2 \mathrm{~cm}$ ! Therefore, $I(v)$ may not be taken out of the integral without approximation.

The major difficulty in evaluating F.y. (1) from experimental data is in the determination of the appropriate lineshape function, $g\left(v-v_{n}\right)$. For situations in which only saturation broadening cccurs, the expected lineshape is Lorentzian. 
while for Doppler broadening the expected lineshape is Gaussian.' However, both broadening mechanisms are operational in our experimental data as shown below.

The saturation parameter is defined as the ratio of the induced transition rate to the spontaneous transition rate:

$$
S=B_{21} I /\left(A_{21}+Q_{21}\right) \text {. }
$$

In Eq. (2), B is the Einstein coefficient for stimulated emission, $I$ is the laser intensity, $A$ is the Einstein coefficient for spontaneous emission, and $Q$ is the quenching rate for the excited state. Unfortunately, $Q$ is not known for $\mathrm{Cu}$ atom in the ${ }^{2} \mathrm{P}$ state, but it should be small, at least at pressures below 5 torr. We assume here that it is zero. For our typical laser conditions $\left(10 \mu\right.$ in a $0.01-\mathrm{cm}^{2}$ spot. 15-ns pulse; $0.1-\mathrm{cm}^{-1}$ bandwidth), one calculates a saturation parameter of 11,500 . That is, the laser strongly saturates the transition. For a lineshape dominated by saturation broadening, the linewidth is given by Eq. (3) where $\Delta v_{n}$ is the natural linewidth for the transition, $\% .3^{n} \times 10^{-4}$ $\mathrm{cm}^{18}$

$$
\left.\Delta v_{s}=\Delta v_{n} \mid \sqrt{ }(1+S)\right]
$$

With $S$ equal to 11,500 , the saturation broadened Lorentzian linewidth in our experiment is calculated to be $0.08 \mathrm{~cm}^{-1}$. This is narrower than the laser bandwidth, and indicates that saturation broadening is not the dominant line broadening mechanism.

Saturation is not the dominant line-broadening mechanism, which is confirmed by fitting lineshape functions to the experimental data, as sho"n for a typical case in Fig. 4. The spectrum was obtained with $S$ torr of he!ium backing gas at a distance of $2.5 \mathrm{~cm}$ from the target. In the upper panel of Fig. 4, a Gaussian lineshape is fitted to the experimental data, while the bottom panel shows a Lorentzian fit to the same data. The lineshape is clearly Griussian and has a half-width oi $1.43 \mathrm{~cm}^{-1}$. The far that the lineshape is Gaussian indicates that saturation broadening, while present. is much less important than Doppler broadening. In addition to produce a linewidih of $1.4 .3 \mathrm{~cm}$ from pure saturation broadening would require a laser energy of approximately $1.5 \mathrm{~mJ} /$ pulse, which is more than our laser system can preduce at this wavelength. Therefore, it is clear that Doppler broadening dominates the miasured linewidth.

The absorption coefficient for a saturated, Doppler broadened transition is given in terms of a constant times a lineshape function called a Voigt profile.' In general, the Voigt profile can not be integrated to give a closed form expression. However, for the limit in which Doppler broadening dominates, the integral can be evaluated exactly and the saturated, inhomogeneously broadened absorption coefficient becomes:

$$
\alpha(v)=\left\{\alpha_{0}\left(v_{0}\right) /[\sqrt{(1+S)}]\right\rangle \exp \left\{\cdot\left[\left(v-v_{0}\right) / \Delta v_{D}\right]^{2}\right\}, \quad(1)
$$

where $\Delta v_{0} / 0.6$ is the Doppler width of the transition. Two realities are evident. First, according to Eq. (4), in the limit of strong Doppler broadening and weak saturation broadening, the lineshape should be Gaussian.' This is the case in all of our data. Second, the absorption coefficient at line center is decreased in the saturation regime by a value of $1 / N(1+S)$. In terms of cross section. Eq. (4) becomes:

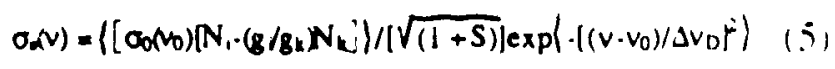

If the excited state population is small at time zero, then Eq. (5) becomes:

$$
\sigma \alpha(v)=\left\langle\left[\sigma \alpha\left(v_{0}\right) N_{i}\right][\sqrt{(1+S)}]\right\rangle \exp \left\{-\left[\left(v-v_{0}\right) /\left.\Delta v_{0}\right|^{2}\right\} .\right.
$$

Inserting Eq. (6) into Eq. (1) for $\alpha_{i k}(v)$ and integrating over $z$, one obtains:

$d l=-N \sigma_{d}\left(v_{0}\right) z /[\sqrt{(1+S)}] \mid \exp \left\{\cdot\left(\left(v-v_{0}\right) /\left.\Delta v_{0}\right|^{2}\right) l(v) d \cdot(7)\right.$

Sirce the laser linewidth, $\Delta v_{1}$, is about a factor of ten less than the Gaussian linewidth, a reasonable approximation is to assume that the laser is a deltil function in frequency space, $l(v)=I \delta\left(v-v_{0}\right)$, and evaluate the integral at $v_{0}$. One is left with the following:

$$
d I / I=-N_{1} \sigma_{0}\left(V_{0}\right) z /[\sqrt{ }(I+S)] .
$$

We analyze the data by fitting each spectrum (1) il Gaussian lineshape function as shown in Fig. 4 . 
The parameters fitted are peak position and halfwidth in wavenumbers, the $100 \%$ transmittance baseline, and the per cent absorption. At line center, the number density is given by $\mathrm{Eq}$. (8). Integrating Eq. (8), one obtains the operative number density equation.

$$
N_{1}=-\left\{\ln \left(L_{0}\right)[V(1+S)]\right\} / \sigma_{0}\left(v_{2}\right) z .
$$

The cross section is given in terms of the Einstein A coefficient by the following:

$$
\sigma=1.5 \times 10^{-1}\left(2 \mathrm{p}^{2} \mathrm{e}^{2} / \mathrm{mc}\right)\left(\mathrm{g}_{\mathrm{k}} / \mathrm{g}_{1}\right) \lambda^{2} \mathrm{~A} .
$$

The cross section for the ${ }^{2} \mathrm{P}_{32}<{ }^{2} \mathrm{~S}_{1 / 2}$ transition at $325.754 \mathrm{~nm}$ is $5.93 \times 10^{16} \mathrm{~cm}^{3 / 2}$. From this value, a calculated saturation parameter, the experimental per cent transmission, and a path length estimated from the size of the visible plume, a numbur density can be calculated. We must assume for the present that the $\mathrm{Cu}$ atoms are uniformly distributed over the path length. This can be confirmed by planar laser-induced fluorescence measurements that will commence in the near future. Typical values for $\mathrm{Cu}$ number densities at 5 torr are $2-4 \times 10^{16} \mathrm{~cm}^{3}$. For comparison, we remove approximately $3 \times 10^{19} \mathrm{Cu}$ atoms per laser shot. This indicates that the atoms removed must be present in a volume considerably smaller than the visible extent of the plume at 5 ton, which is approximately $8 \mathrm{~cm}^{3}$. This is certainly possible as shown below by the $\mathrm{Cu}$ atom time-ofthight data.

\section{Cu Atornic Velocity by Time oí Flight}

Figure 5 shows a series of curves whose amplitude is proporional to the nuinber density of $\mathrm{Cu}$ atom at different distances from the target. als a function of delay time between the ablation lisier pulse and the LIF probe laser pulse. Thie curves pictured in Fig. 5 were obtained under vacuum conditions ( $-10 \mathrm{mtorr})$. Dividing the distance travelled between two curves by the delay time difference gives a velocity. The velocities measured from Fig. 5 are approximutely $2 \times 10^{6} \mathrm{~cm} / \mathrm{s}$.

In Fig. 5, the $\mathrm{Cu}$ atom density peaks at short delay times of $2-5 \mu 5$, but the signal persists at a level perhaps $20 \%$ of the peak value for times in excess of 50 $\mu \mathrm{s}$. The data indicate that the majority of the $\mathrm{Cu}$ atoms are present in a spheric:al shell that is expanding quickly outward. This is the prediction of blast wave theory as developed by Zel' dovich and Rayzer.'

The situation with 5 torr of He backing gas is noticeably different. Time-of-flight curves for this condition are shown in Fig. 6. It is apparent that the backing gas slows and broadens the timeof-flight disuribution considerably when compared to the low-pressure data. The average velocity of the $\mathrm{Cu}$ atoms $0.5 \mathrm{~cm}$ from the target surface is $4.4 \times 10^{5} \mathrm{~cm} / \mathrm{s}$ and decreases to $1.8 \times 10^{3} \mathrm{~cm} / \mathrm{s}$ $3.2 \mathrm{~cm}$ from the target. This trend continues when the pressure is increased to 50 torr. The velocity decreases to approximately $2 \times 10^{3} \mathrm{~cm} / \mathrm{s}$ only $0.7 \mathrm{~cm}$ from the target.

\section{Cu, LIF Detection and Temperature Measurement}

Figure $i$ is a fluorescence excitation scan of the $\mathrm{Cu}_{2} \mathrm{~A} \cdot \mathrm{X}(u, \eta)$ band taken with 50 torr of background He gas. 'The probe laser-target distance was $0.6 \mathrm{~cm}$ in this case. The signal instantaneously disappears in the absence of the ablation laser pulse indicating that the $\mathrm{Cu}_{2}$ is formed on a single shot within the plume. The A-X system of $\mathrm{Cu}_{2}$ has been thoroughly studied previously by a number of groups, but most recently by Page, Gudeman, and Mitchell.' Rotational constants have been determined for both states and. therefore, rotational temperatures can be obtuined by simulating the rovibronic band contours. Such a simulation of the $\mathrm{Cu}_{2} \mathrm{~A}-\mathrm{X}$ band $(0,0)$ band at $300 \mathrm{~K}$ is given in Fig. 8. The simulation does $\mathrm{nox}$ include features due to the $(1,1)$ band, which are seen in the experimental spectrum of Fig. 7. The simulation does not adequately reflect the experi. mental spectrum, primarily because the bandhe:ad is not intense enough relative to the rotitional structure in the 491.0-10 492.0-nm region. Bused on simulations at higher temperatures, we believe that this is an experimental artifact due to some combination of detection electronics saturation and r.on-uniform monochromator spectral response. Both problems are being remedied. However, it is apparent that the lemperature in the experimental scan is relatively low, being in the neighborhood of $300 \mathrm{~K}$. 
In principle, the ratio of the $(0,0)$ and $(1,1)$ bandhead intensities can be used to calculate a vibrational temperature assuming a Boltzmann distribution in the ground-state $\mathrm{Cu}$ vibrational distribution. This iemperature is also approximately $300 \mathrm{~K}$ based on the spectrum of Fig. 7; however, it is subject to the same experimental biases as discussed for the rotational temperature above. The fact that these temperatures are quite low seems somewhat surprising; however, at room temperature the collision frequency for 50) torr of He is $3 \times 10^{2} \mathrm{~s}^{-1}$, and it is most likely some what higher in our experiment. After a $\mathrm{Cu}_{2}$ molecule is formed, it undergoes a collision approximately every $33 \mathrm{~ns}$; after several such collisions it will be thermalized. Therefore, it is not surprising that we observe room temperature $\mathrm{Cu}_{2}$ with 50 tort of background gas. In order to observe the nascent temperature, one must use lower pressures of backing gas.

Figure 9 shows time-of-flight curves for $\mathrm{Cu}_{2}$ with 50 torr of He backing gas obtained in the same manner as those for $\mathrm{Cu}$ in Figs. 5 and 6. The relative signal intensities for each position are approximately correct. Two realities are noteworthy. First, the $\mathrm{Cu}_{2}$ is moving extremely slowly under these conditions. This is not particularly surprising considering the collision frequency. Second, although there appears to be a higher number density of $\mathrm{Cu}_{2}$ at $\mathrm{z}=2.5 \mathrm{~cm}$ from the relative signal strengths, one must also consider the effect of temperature on the signal. The laser excited the bandhead in each of the three curves of Fig. 9. The bandhead in the $\mathrm{Cu}_{2} \mathrm{~A}-\mathrm{X}$ system occurs in the R-branch at relatively low J $(\mathrm{J}=16)$. At low temperature, most of the intensity accumulates in the bandhead, while at high temperature the bandhead is diminished in intensity. Therefore, in order to make a direct comparison of relative $\mathrm{Cu}_{2}$ number density, one must know the temperature as well as the signal strength on the bandhead.

\section{Rayleigh Scattering from the Particulate}

In addition, we have observed Rayleigh scattering from the condensate formed in the plasma plume with 50 corr of helium background gas. The signal instantaneously disappears in the abscence of the ablation laser pulse indicating that the pariculate is formed on a single shot within the plasma plume. The Rayleigh-scattered signal was observed to be a factor of $2-3$ stronger than the $\mathrm{Cu}_{2} \mathrm{LIF}$ signal when resonance fluorescence is detected. The strengih of the Rayleigh signal is somewhat surprising considering the small size $(\leq 150 \mathrm{~nm})^{10}$ of the particles relative to the 490.3 . nm pump wavelength. This signal can be used to measure the location and velocity of the particles.

\section{Conclusions and Furure Directions}

We have developed diagnostics for $\mathrm{Cu}$ nunnber density and velocity as well as for $\mathrm{Cu}_{2}$ detection, velocity, and temperature in laser-ablated copper plasmas. These diagnostics allow us to measure very high $\mathrm{Cu}$ velocities $\left(2 \times 10^{6} \mathrm{~cm} / \mathrm{s}\right)$ in the absence of any added backing gas as well is the effect on velocity of a backing gas. Copper atom densities are as high as $2-4 \times 10^{16} \mathrm{~cm}^{-3}$ witi 5 torr of He background gas assuming a uniform distribution of $\mathrm{Cu}$ atoms across the plume. Vibrational and rotational temperatures for $\mathrm{Cu}_{2}$ with 50 torr of He backing gas are approximately $300 \mathrm{~K}, 0.6 \mathrm{~cm}$ from the target surface, but further development of the temperature diagnostic is necessary to refine the measurement. The temperature is necessary to place the $\mathrm{Cu}_{2}$ LIF signal (number density) on a relative scale because of the effect of temperature on the intensity of the bandhead. Rayleigh scattering from the particulate is quite strong at $\mathbf{5 0}$ torr. and this signal can be used to measure the location and velocity of the paricles. The LIF signal from $\mathrm{Cu}_{2}$ and the Rayleigh-scattered signal from the paricles instantaneously disappears in the absence of the ab. lation laser pulse indicating that the particles are formed on a single laser shot. Furure experiments will obtain two-dimensional images of $\mathrm{Cu}$ atoms. $\mathrm{Cu}_{2}$, and particulate in an atempt to correlate the disappearance of $\mathrm{Cu}$ atom with the appearance of $\mathrm{Cu}_{2}$ and ultimately particulate. In addition, we intend to search for $\mathrm{Cu}_{3}$ via LIF diagnostics.

\section{References}

1. E. A. Rohlfing and J. J. Valentini, J. Chem. Phys. 84, 6560 (1986). 
2. See for example, X. D. Wu, R. E. Muenchausen, S. Foltyn, R. C. Estler, R. C. Dye C. Flamme, N. S. Nogar, A. R. Garcia, J. Martin, and J. Tesmer, Appl. Phys. Leit. 56. 1481 (1990).

3. J. L. Lyman, Synthesis of Materials with Infrared and Ultraviolet Lasers, SPIE Proceedings \# 1033 International Conference on Trends in Quantum Electronics, Central Institute of Physics, Bucharest, Romania (1988).

4. D. E. Powers, S. G. Hansen, M. E. Geusic, D. L. Michalopoulos, and R. E. Smalley, J. Chem. Phys. 78, 2866 (1983).

5. B. H. Weiller, P. S. Bechthold, E. K. Parks, L. G. Pobo, and S. J Riley, J. Chem. Phys. 91. 4714 (1989).

6. F. W. Froben, J. Kolenda, and K. Moller, J. Phys. D 12, 485 (1989).

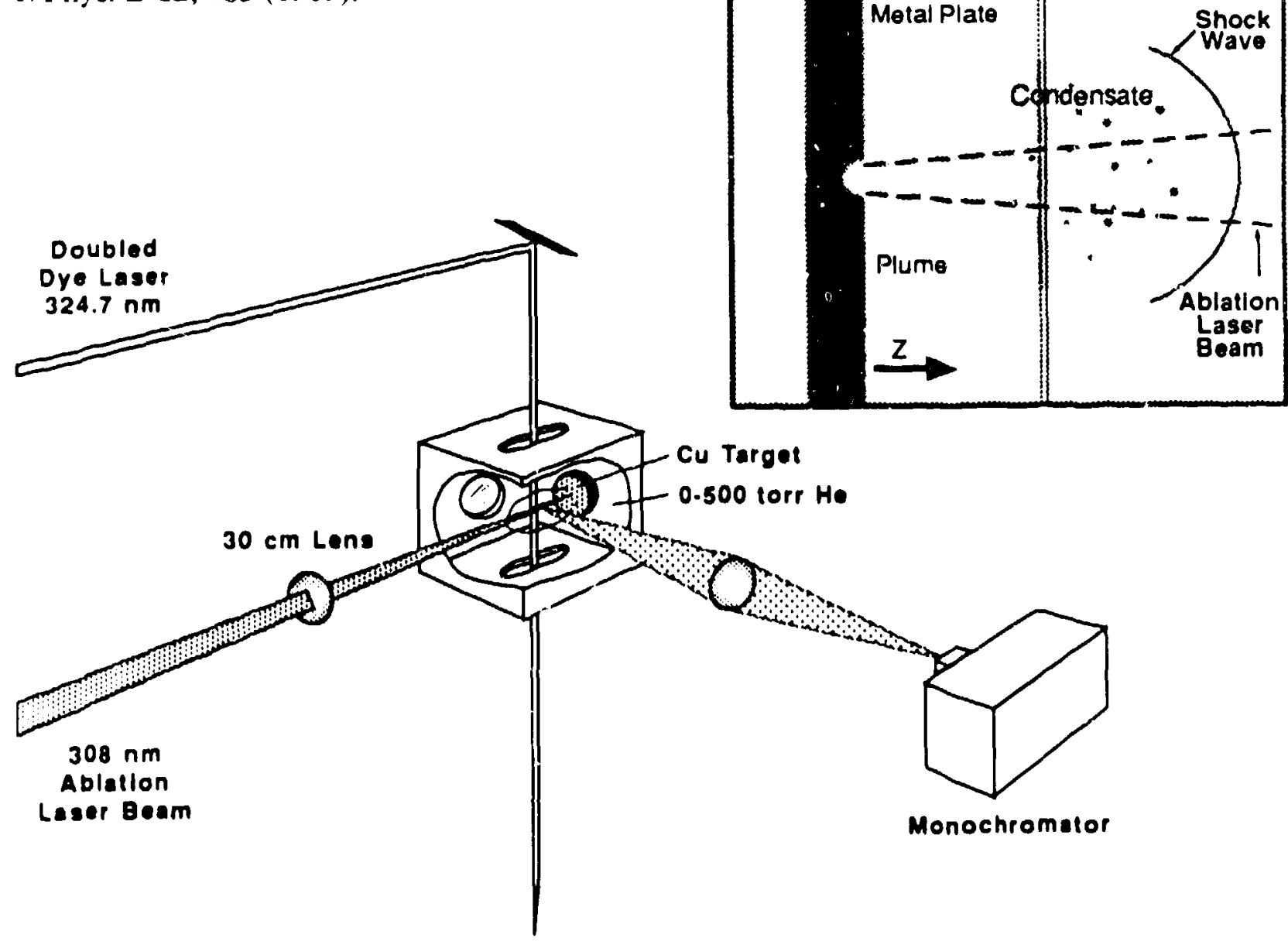

7. R. H. Page and C. S. Gudeman. J. Chem. Phys. 94, 39 (1991).

8. W. Demtroder, Laser Spectroscopy: Bas!c Concepts and Instrumentation, Springer Series in Chemical Physics 5, SpringerVerlag Berlin (1981).

9. Zel'dovich, Ya. B. and Rayzer, Yu. P., Phys. ics of Shock Waves and High Temperature Gas Dynamic Phencmena, Moscow Nauka (1966).

10. A. D. Sappey, T. K. Gamble, P. J. Wantuck, H. H. Watanabe, and B. Benjamin, Diagnostic Studies of Laser Ablated Iron . :asmas. Los Alamos Unclassified Repon $90-3258$ (1990).

Fig. 1. Sihematii diagram of Laser-Induced Fluorescence Apparatus. Inset shows details of the ablatiın plume and the probe laser beam. 


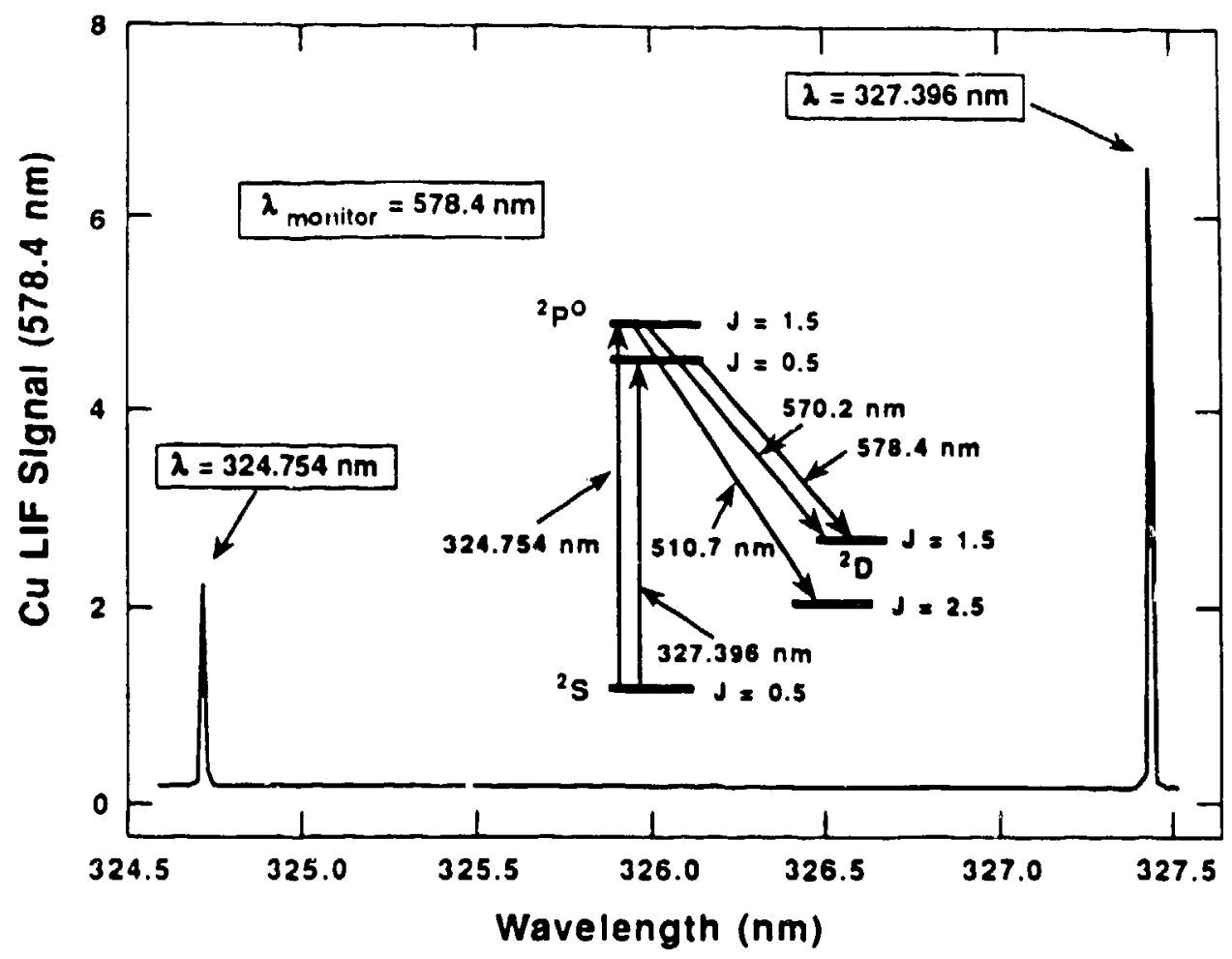

Fig. 2. Laser-induced fluorescence scan of the $C u$ atom ${ }^{2} P^{0} \leftarrow{ }^{2} S$ transitions. Fluorescence is detected at $578.4 \mathrm{~nm}$. The probe laser is located $2.5 \mathrm{~cm}$ from the surface and the backing gas pressure is 5 torr He. The delay between the lasers is $500 \mu \mathrm{s}$.

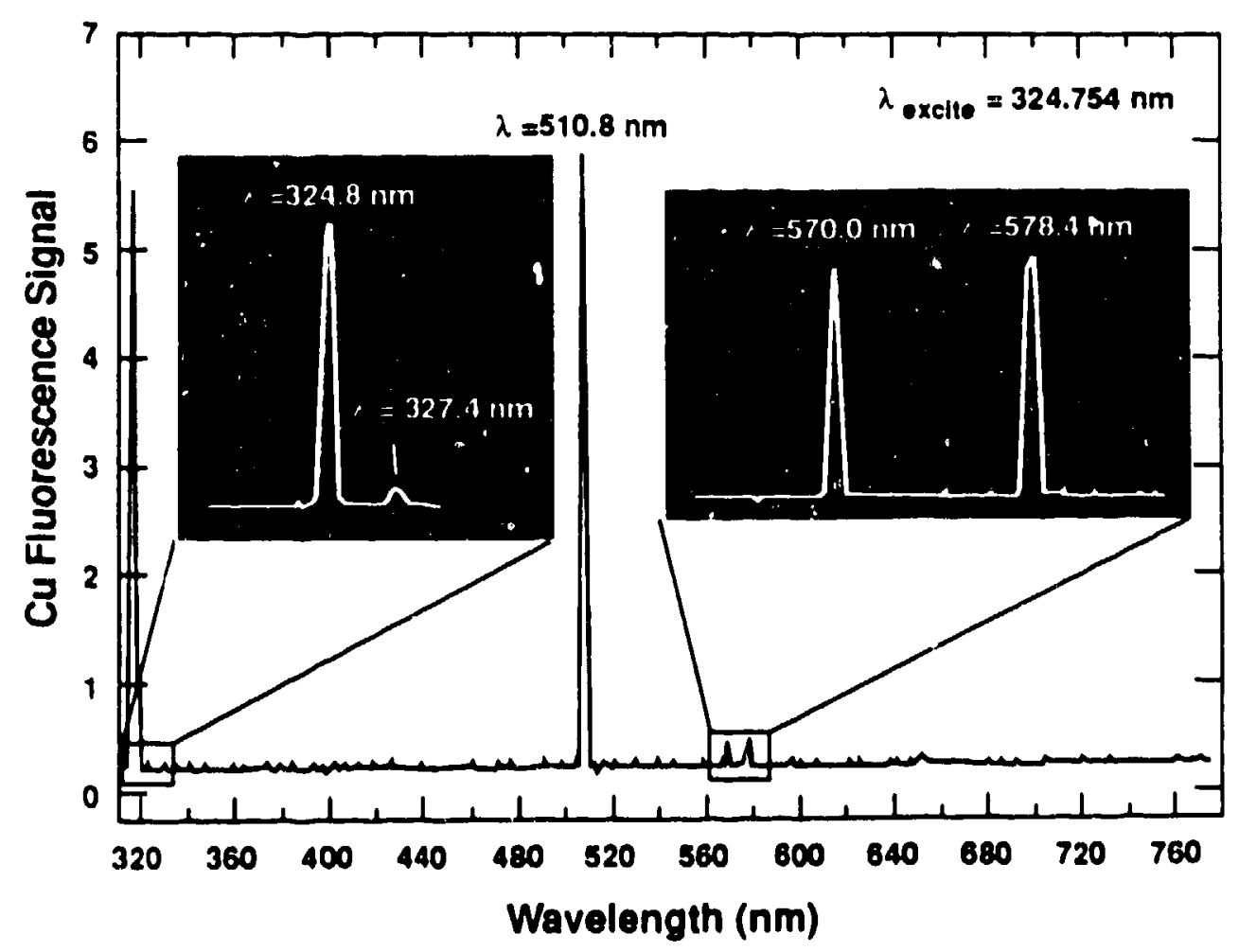

Fis .1. Dispersed fluorescence scan of $\mathrm{Cu}$ atom exciting at $324.75 \mathrm{~nm}$. The fact that we observe fluorescencic at $327.4 \mathrm{~nm}$ and $578.4 \mathrm{~nm}$ indicates that energy transfer occurs quickly in the ${ }^{2} P$, manifold. The spectrum was obtained under the same conditions as the excitation specirum of Fig. 2. 


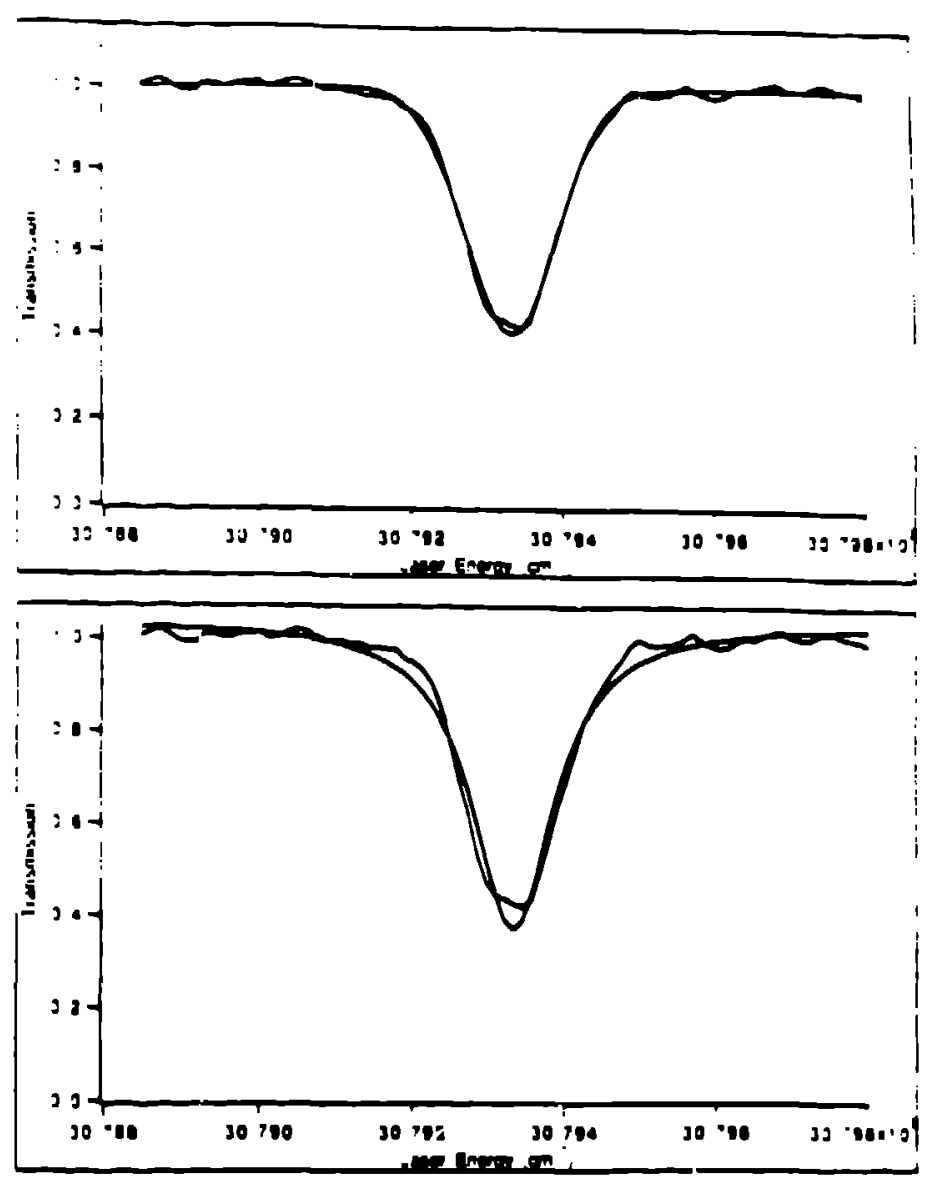

Fi: \& Top panel: Gaussian fit to experimental absorption spectrum. The spectrum was obtained under thi sume conditions as the LIF spectrum of Fig. 2. The half-width of the fit is $1.43 \mathrm{~cm}^{\prime \prime}$.

Buttum panel: Lorentzian fit to the same experimental absorption spectrum. Clearly the Gaussian fit ts better indicating that Doppler broadening is the dominant broadering mechanism.

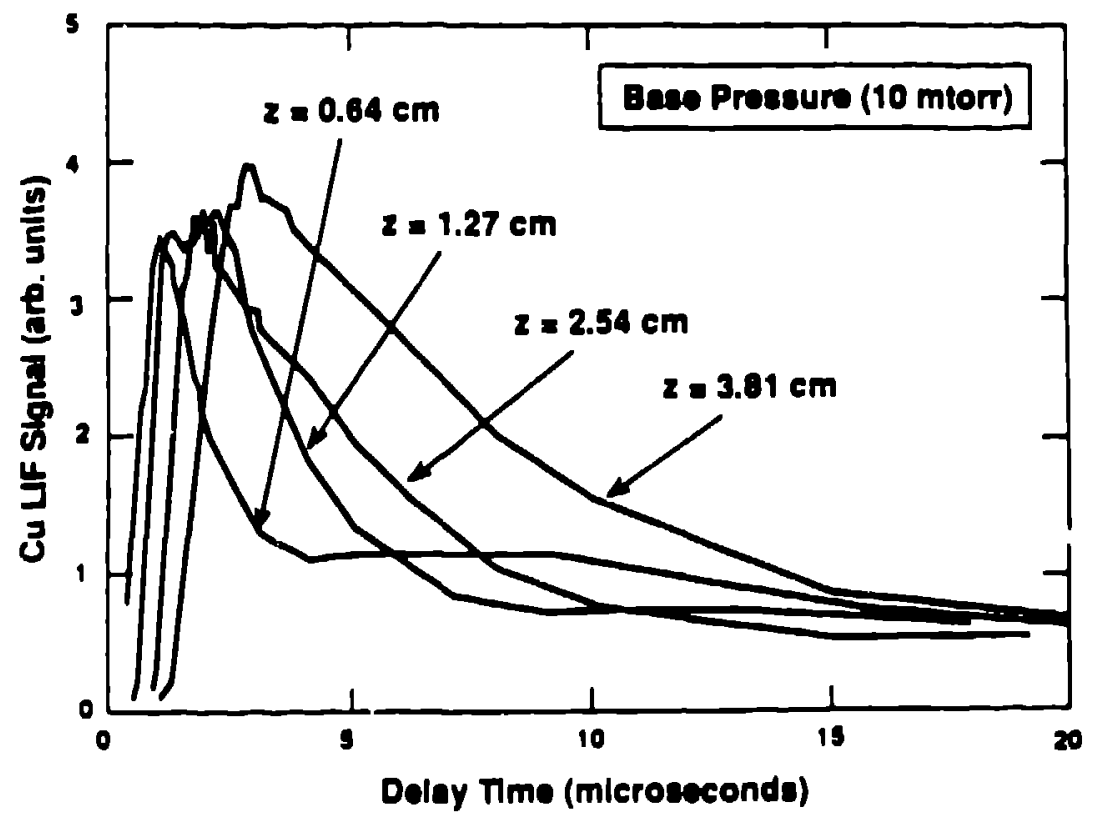

F.: (¿ LIF time-off-flight curves obtuined with 10 merr of He backing gas. The curves can be used to me'ssure $C u$ velocity thas is $\sim 2 \times 10^{6} \mathrm{c}: \mathrm{w} / \mathrm{s}$ under these conditions. 


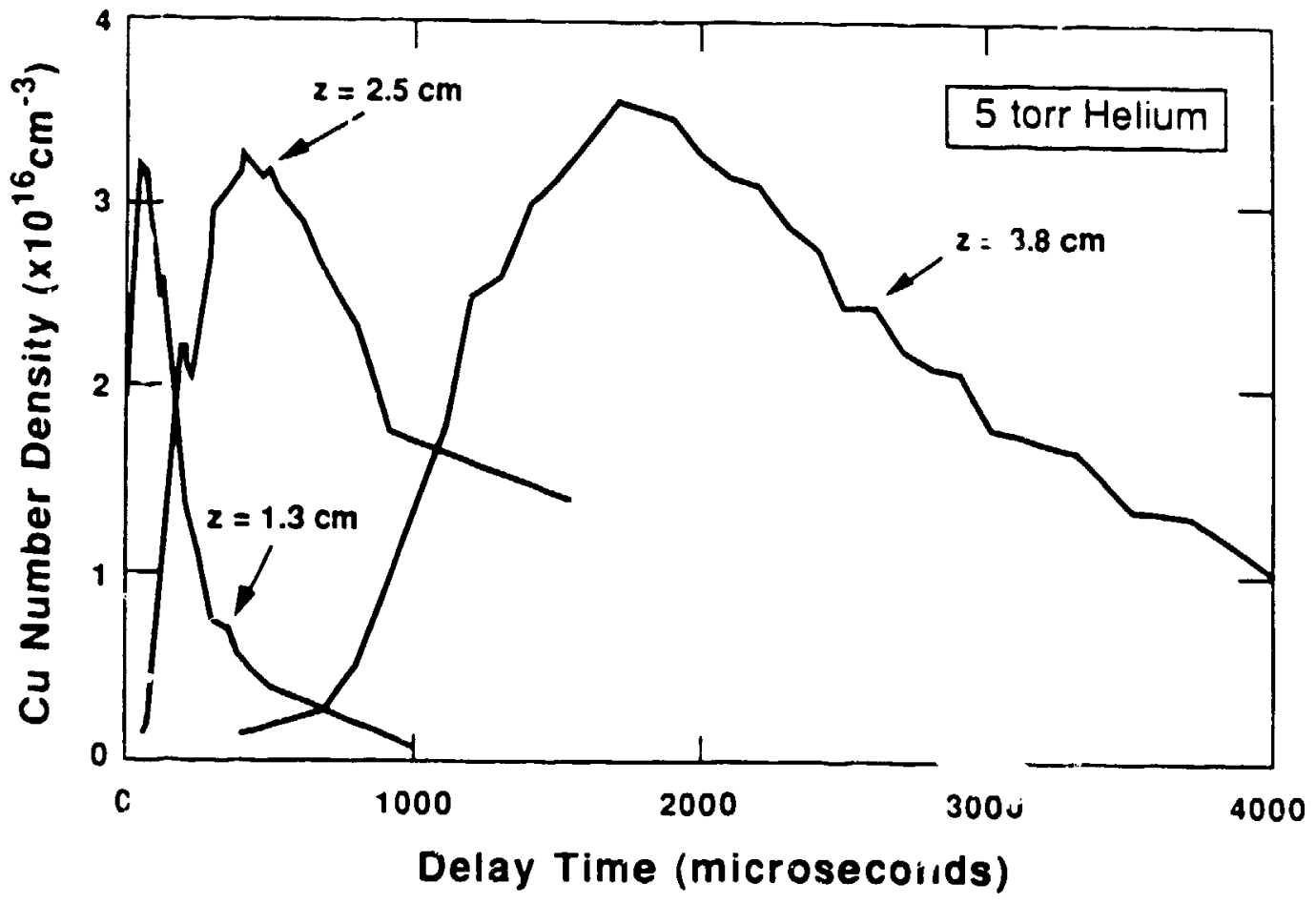

Fig. 6. Cu atom time-of-flight curves with 5 torr He backing gas. The He slows and broadens the time-offlight curves when compared with those taken at low pressure (Fig. 5).

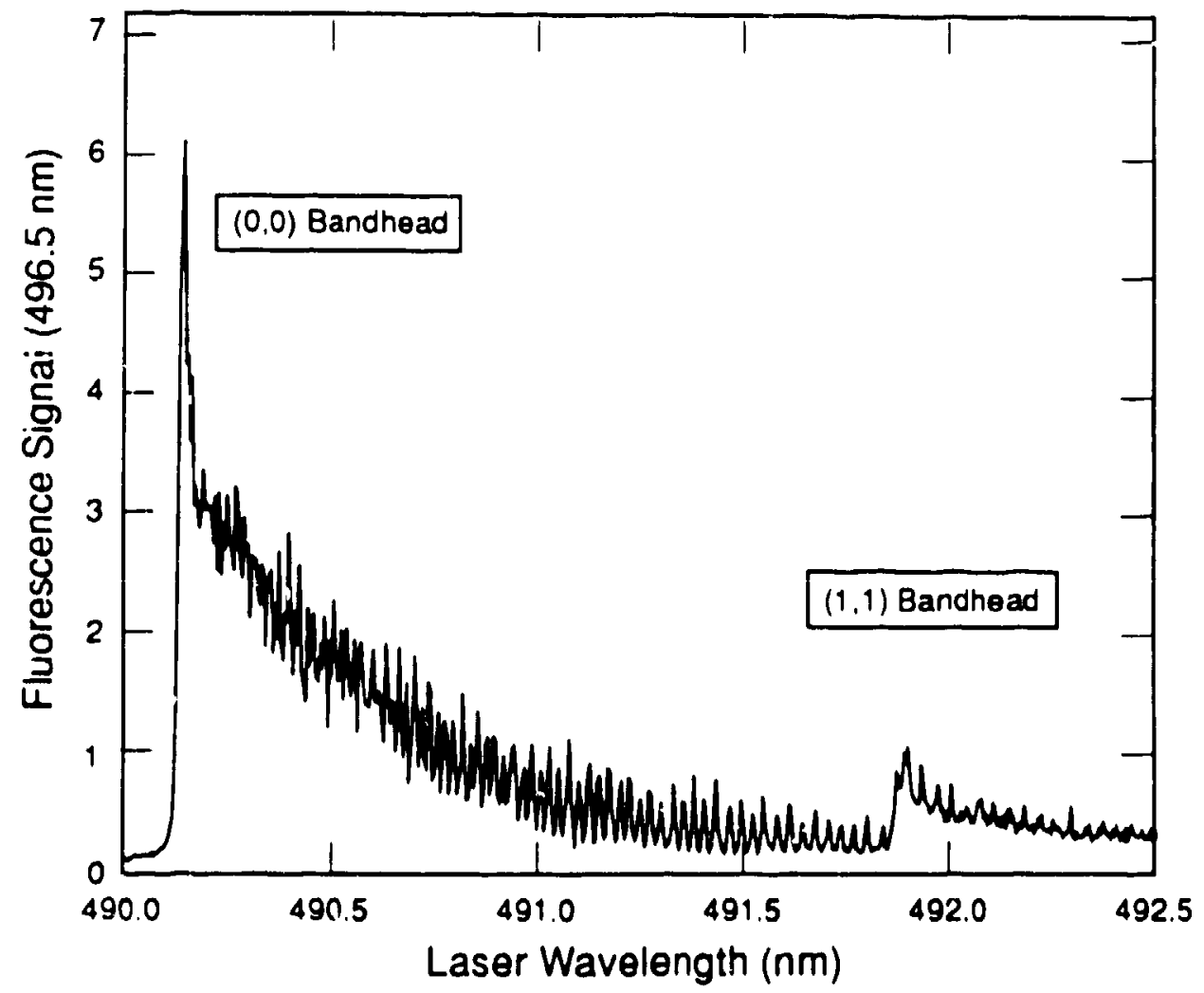

Fig. 7. LF excitation scan of the $C u_{2} A-X(0,0)$ band. Fluorescence is detected on the $(0.1)$ band at $496.7 \mathrm{~nm}$. The rotational structure can be simulated to obtain a temperature assuming a thermal distribution in the ground state. A vibrational temperature is obtained from the ratio of the $(0,0): 12 d(1,1)$ bandhects 


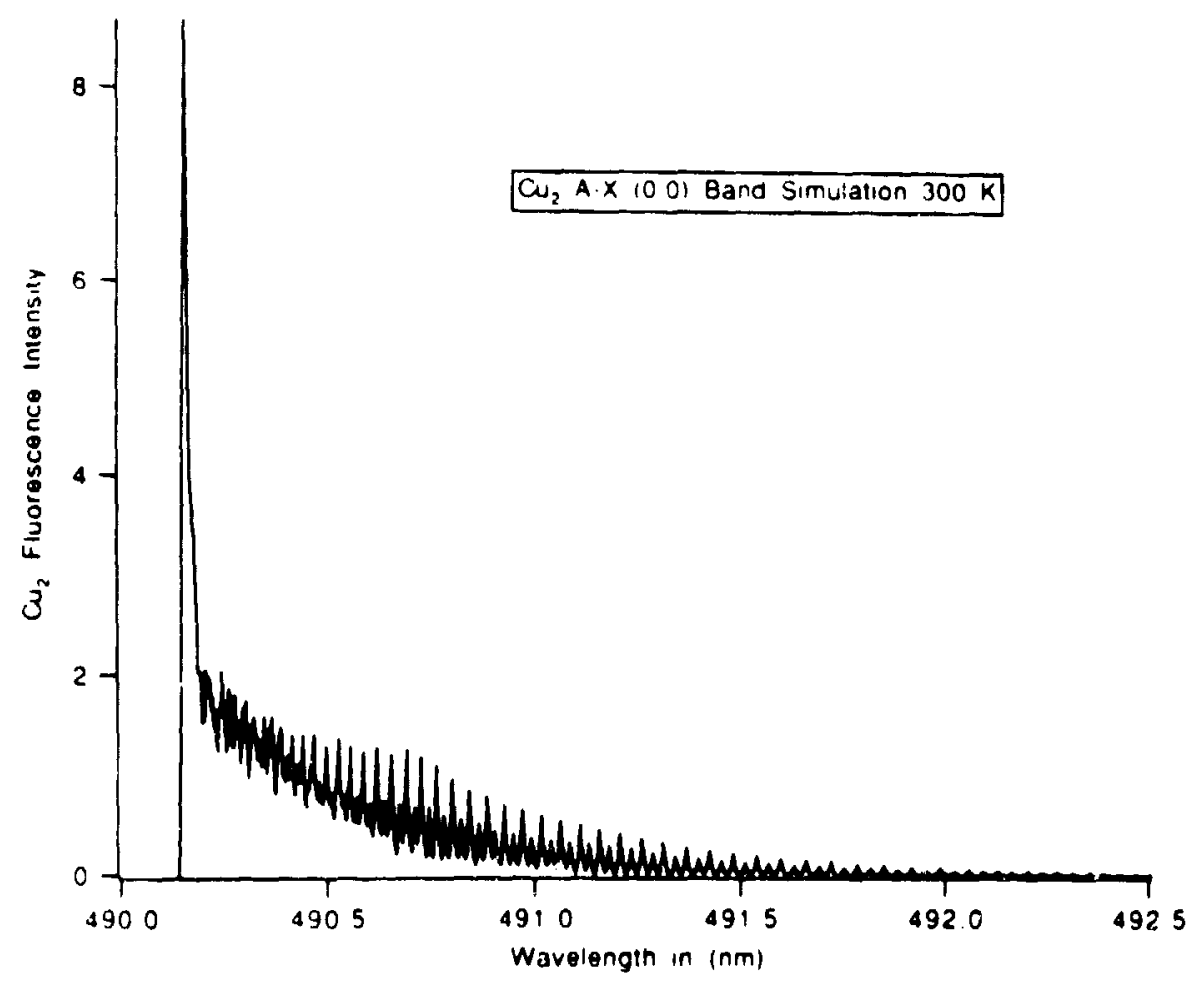

Fig. 8. Compuser simulation of the $\mathrm{Cu} 2 \mathrm{~A} \cdot \mathrm{X}(0,0)$ band at $300 \mathrm{~K}$. The $(1,1)$ band is not included in the simulli. tion.

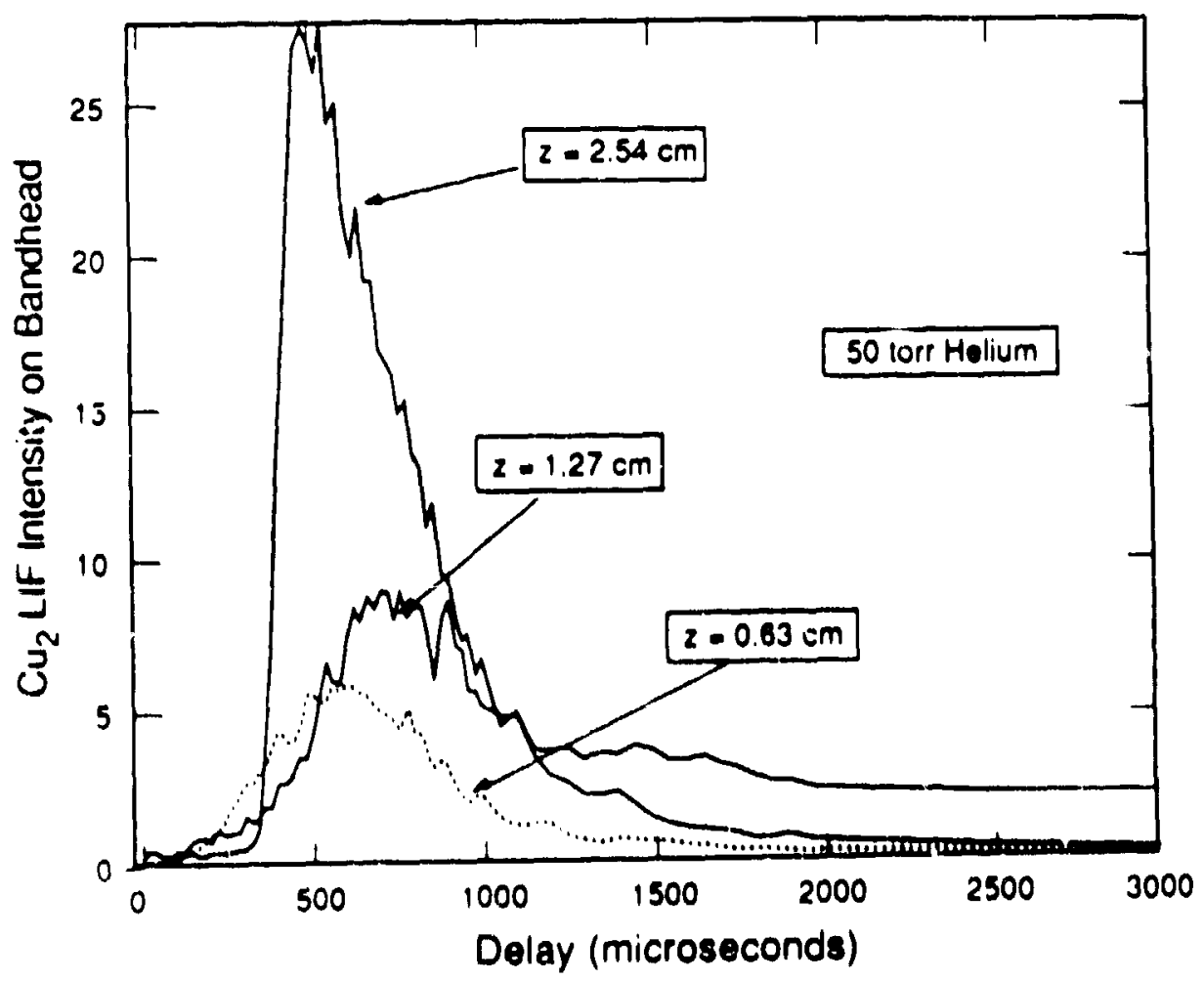

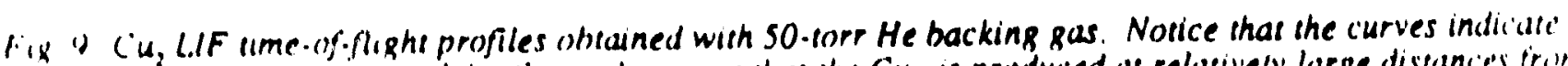
biry slow movement if the $C u_{2}$ and suggess that the $C u$, is produced at relatively large distances' from the lurget 